\title{
Multiple Bases of Human Intelligence Revealed by Cortical Thickness and Neural Activation
}

\author{
Yu Yong Choi, ${ }^{1,2}$ Noah A. Shamosh, ${ }^{4}$ Sun Hee Cho, ${ }^{3}$ Colin G. DeYoung, ${ }^{4}$ Min Joo Lee, ${ }^{1}$ Jong-Min Lee, ${ }^{6}$ Sun I. Kim, ${ }^{6}$ \\ Zang-Hee Cho, ${ }^{7}$ Kyungjin Kim, ${ }^{1}$ Jeremy R. Gray, ${ }^{4,5}$ and Kun Ho Lee ${ }^{1}$ \\ ${ }^{1}$ School of Biological Sciences, ${ }^{2}$ Interdisciplinary Program in Brain Science, and ${ }^{3}$ Department of Biology Education, Seoul National University, Seoul 151- \\ 742, Korea, ${ }^{4}$ Department of Psychology and ${ }^{5}$ Interdepartmental Neuroscience Program, Yale University, New Haven, Connecticut 05620, ${ }^{6}$ Department of \\ Biomedical Engineering, Hanyang University, Seoul 133-605, Korea, and 7Neuroscience Research Institute, Gachon Medical School, Incheon, Seoul 450- \\ 760 , Korea
}

We hypothesized that individual differences in intelligence (Spearman's $g$ ) are supported by multiple brain regions, and in particular that fluid $(g F)$ and crystallized $(g C)$ components of intelligence are related to brain function and structure with a distinct profile of association across brain regions. In 225 healthy young adults scanned with structural and functional magnetic resonance imaging sequences, regions of interest (ROIs) were defined on the basis of a correlation between $g$ and either brain structure or brain function. In these ROIs, $g C$ was more strongly related to structure (cortical thickness) than function, whereas $g F$ was more strongly related to function (blood oxygenation level-dependent signal during reasoning) than structure. We further validated this finding by generating a neurometric prediction model of intelligence quotient (IQ) that explained $50 \%$ of variance in IQ in an independent sample. The data compel a nuanced view of the neurobiology of intelligence, providing the most persuasive evidence to date for theories emphasizing multiple distributed brain regions differing in function.

Key words: general intelligence; crystallized intelligence; fluid intelligence; IQ; cortical thickness; fMRI

\section{Introduction}

People's performance on a given task tends to covary positively with their performance on a wide variety of measures. Spearman (1904) proposed that this positive manifold was attributable to a single underlying cause, general mental ability $(g)$, an interpretation that has proven to be much more difficult to establish than the correlations themselves (Chabris, 2006). To test Spearman's hypothesis, Duncan et al. (2000) used positron emission tomography to image 13 subjects performing relatively difficult, high- $g$ tasks and relatively easy, low- $g$ tasks. Spearman's unitary view of the mechanisms of $g$ was taken to predict a single locus of activation, whereas the competing, nonunitary view of Thurstone (1940) was taken to predict distributed activation. Across three difficult tasks, compared with a control task, only lateral prefrontal cortex (PFC) was activated in all three comparisons. Despite the conceptual elegance of this finding, subsequent studies assessing individual differences in intelligence have implicated a much wider network of regions, using several techniques (Gray and

Received July 13, 2008; revised Aug. 4, 2008; accepted Sept. 3, 2008.

This work was supported by the Brain Research Center of the 21st Century Frontier Program in Neuroscience and the Biotool R\&D Project for Cell Research from the Korean Ministry of Science and Technology. K.H.L. was supported by the BK 21 program from the Korea Ministry of Education. J.R.G. was supported by National Science Foundation DRL CAREER 0644131.

Correspondence should be addressed to either of the following: Dr. Kun Ho Lee, School of Biological Sciences, Seoul National University, Seoul 151-742, Korea, E-mail: leekho@snu.ac.kr; or Dr. Jeremy R. Gray, Department of Psychology and Interdepartmental Neuroscience Program, Yale University, New Haven, CT 05620, E-mail: jeremy.gray@yale.edu.

DOI:10.1523/JNEUROSCI.3259-08.2008

Copyright $\odot 2008$ Society for Neuroscience $\quad$ 0270-6474/08/2810323-07\$15.00/0
Thompson, 2004), including functional imaging in tandem with mediation analyses (Gray et al., 2003) and the method of correlated vectors (Lee et al., 2006), studies of lesion patients (Tranel et al., 2008), and multivariate molecular genetic measures (Kovas and Plomin, 2006). A critical but unasked question is whether different regions contribute to $g$ as functionally distinct units, each making some unique contribution, or whether the regions are all part of a single functionally unified (but spatially distributed) network.

One way to test for separable neural contributions to $g$ is to investigate differences at a lower level in the ability hierarchy. Cattell (1943) distinguished between general crystallized $(g C)$ and fluid $(g F)$ intelligence when assessing the neural basis of intelligence. $g C$, sometimes described as verbal ability, is more dependent on accumulated knowledge in long-term storage, including semantic memory. $g F$ refers to reasoning ability, and is known to depend on working memory (Engle et al., 1999). Although $g C$ and $g F$ are typically correlated and can be considered subfactors of $g$ (Jensen, 1998), they are conceptually and empirically separable. For instance, $g C$ continues to increase over the lifespan, but $g F$ peaks in early adulthood and then declines (Craik and Bialystok, 2006). Furthermore, at the neural level, lesion studies demonstrated that patients with anterior temporal damages perform poorly on tests of semantic knowledge, whereas prefrontal patients typically show profound deficits in solving diverse reasoning tasks (Duncan et al., 1996; Waltz et al., 1999).

To show that multiple brain areas contribute in distinct ways to individual differences in $g$, we tested the more specific hypoth- 
esis that $g$ can be fractionated into separable biological substrates for $g C$ and $g F$. Although this could be tested in many ways, we focused on differences in brain structure and function, because both have been correlated with cognitive abilities: vocabulary learning induces changes in gray matter density (Lee et al., 2007), and reasoning ability covaries with activation level (Lee et al., 2006). Our results revealed that in $g$-related regions, $g C$ and $g F$ relate differentially to gray matter thickness and functional brain activity. Thus, an additional goal was to probe the robustness of our findings by generating and validating a neurometric intelligence quotient (IQ) model of $g$, i.e., to predict $g$ prospectively from brain structure and function. By investigating neural correlates of intelligence at both the structural and functional level, our work goes beyond extant neuroimaging studies of human intelligence, which have sought to correlate $g$, $g C$, or $g F$ with either structural (Gong et al., 2005; Shaw et al., 2006) or functional (Gray et al., 2003; Lee et al., 2006) measures, but not both.

\section{Materials and Methods}

Participants. Protocols were approved by the relevant institutional review boards (Seoul National University, Catholic University of Korea), and written informed consent was obtained from participants. We administered two reliable and valid intelligence tests, the Wechsler Adult Intelligence Scale-Revised (WAIS) and the Raven's Advanced Progressive Matrices Set II (RPM), to assess psychometric IQ and fluid reasoning ability, respectively (Carroll, 1993), in 408 healthy young adults. A subset of participants were selected for the imaging experiments $(n=225)$ to provide an approximately even distribution within a broad range of WAIS full-scale IQ (FSIQ) $(81 \leq \mathrm{IQ} \leq 150): 122$ male/103 female; IQ $($ mean $\pm S D)=119 \pm 15 ; R P M$ score $=28.1 \pm 6.0$ (for further information about subjects, see supplemental Methods and supplemental Table 1, available at www.jneurosci.org as supplemental material). They were sampled within a narrow age range of young adults $(20.9 \pm 2.9$ years), to avoid developmental or aging periods in which the brain changes radically (Sowell et al., 2003; Shaw et al., 2006).

Magnetic resonance imaging acquisition and analysis. One hundred sixty-four and one hundred nine subjects were scanned for anatomical and functional magnetic resonance imaging (MRI) data, respectively, and forty-eight of these subjects contributed both structural and functional samples. Details of the imaging acquisition and the functional MRI tasks can be found in supplemental Methods (available at www. jneurosci.org as supplemental material). From the high-resolution, T1weighted brain scans, triangular mesh models of white and gray matter surfaces were automatically reconstructed in native space by the CLASP (constrained Laplacian-based automated segmentation with proximities) algorithm. Cortical thickness was measured at each of 81,920 points throughout the cerebral cortex, by calculating the euclidean distance between linked vertices on the inner and outer cortical surface meshes. The statistical parameters for cortical activation were acquired using the statistical parametric mapping software (SPM2). Further analysis methods can be found in supplemental Methods (available at www. jneurosci.org as supplemental material).

Neurometric IQ model. We used a two-stage approach to developing a model for estimating IQ scores from brain images. We first specified a prediction model, and then tested it in a statistically independent sample to avoid conceptual circularity. To specify a prediction model, we used structural images from 116 participants with high-resolution structural MR images, from which we estimated brain volume and the thickness of cortical gray matter. We used functional data from 61 different participants with functional MR images acquired as they performed reasoning and control tasks. Brain volume (gray matter + white matter) was a potential predictor a priori. Regions of interest (ROIs) were defined entirely on the basis of the dataset for model specification (see Fig. $4 A$; supplemental Fig. 5, available at www.jneurosci.org as supplemental material). To reduce redundancy (collinearity), we averaged ROIs into a single predictor if they were significantly correlated with each other $(p<$ $0.001): s_{1}=(\mathrm{ATC}+\mathrm{OTC}+\mathrm{MTC}) / 3, s_{2}=\mathrm{LPC}$, and $f=(\mathrm{ACC}+\mathrm{LPFC}$
$+\mathrm{RPFC}+\mathrm{LPPC}+\mathrm{RPPC} / 5$, where ATC is anterior temporal cortex, OTC is opercular temporal cortex, MTC is medial temporal cortex, LPC is lateral parietal cortex, ACC is anterior cingulate cortex, LPFC is left prefrontal cortex, RPFC is right prefrontal cortex, LPPC is left posterior parietal cortex, and RPPC is right posterior parietal cortex. During the model specification stage, we did not have datasets with both structural and functional images, and so we were forced to obtain the constants from two separate stepwise regressions: IQ on volume (vol), $s_{1}, s_{2}$, and vol $\times$ sex (structural sample), and IQ on $f$ (functional sample). All predictor-by-sex interaction terms except vol $\times$ sex did not explain variance in IQ. Sex was coded female $=0$, male $=1$; and $f$ was standardized.

The regression equation from the structural sample (structural IQ model; $R=0.64$ ) was given as follows: $\mathrm{IQ}=25+0.000045 \mathrm{vol}+23 s_{1}-$ $19 s_{2}-0.0000044 \mathrm{vol} \times$ sex. The regression equation from the functional sample (functional IQ model; $R=0.55$ ) was given as follows: IQ $=118+$ 9.2f. Finally, the neurometric IQ model combining the two equations above was written in general form (with free parameters $\alpha$ and five $\beta$ parameters) as follows: IQ $=\alpha+\beta_{1} \times$ vol $+\beta_{2} \times s_{1}+\beta_{3} \times s_{2}+\beta_{4} \times$ $\operatorname{vol} \times \operatorname{sex}+\beta_{5} \times f$.

To test the model, we acquired IQ, structural, and functional data from 48 independent participants from the same population (see Fig. $4 B$ ). This sample for test equaled the samples for model specification in both FSIQ and RPM scores (all $p$ values $>0.65$, one-way ANOVA). Values were extracted from the ROIs and averaged into $s_{1}, s_{2}$, and $f$. We estimated IQ in two ways, first by fitting the model using regression in the test sample (to fix the $\alpha$ and $\beta$ parameters in the equation above) and using the strict model (no free parameters, in the next equation, below). The strict model, using constants derived from the modeling datasets, was written as follows: IQ $=25+0.000045 \mathrm{vol}+23 s_{1}-19 s_{2}-$ $0.0000044 \mathrm{vol} \times \operatorname{sex}+9.2 f$.

\section{Results}

\section{Intelligence-related regions: structural versus functional correlates}

We first tested for associations of $g$ with brain structure and function. As expected, brain volume correlated with IQ $(r=0.35 ; p<$ $0.001)$. The thickness of the gray matter of the cerebral cortex was determined using an advanced, automated surface extraction technique (Kim et al., 2005) in a large sample $(n=164)$. Across brain regions, there were substantial fluctuations in the correlation between cortical gray matter thickness and WAIS FSIQ $(p<$ 0.05 ) (Fig. $1 A$ ). The correlation map with FSIQ revealed a striking asymmetry in favor of the left hemisphere (Fig. 1C,D). All positive correlates $(p<0.001)$, including the ATC, OTC, and inferior temporal cortex (ITC), converged on the temporal lobe, primarily on the left side and its adjacent areas, whereas a negative correlation was found only in the lateral parietal cortex $(p<$ $0.001)$. Given our intentionally narrow age range, it is not surprising that age was not associated with the cortical thickness throughout the cerebrum $(p<0.001)$.

Using the same statistical criteria, we investigated the relationship between intellectual ability and neural activity of the cortical network during a fluid reasoning task $(n=109$; see Materials and Methods). There were widely distributed positive correlations throughout the entire brain and no significant negative correlates (Fig. $1 B$ ). In contrast to the structural correlates, the functional correlates had no clear lateralization (Fig. 1E,F). With RPM score as a covariate, the overall patterns of both the structural and the functional maps were generally similar to those derived from WAIS IQ (supplemental Fig. 1, supplemental Table 2, available at www.jneurosci.org as supplemental material). In a comparison between the structural maps, RPM scores tended to be correlated more strongly with cortical thickness in the lateral and medial prefrontal regions than were WAIS scores $(p<0.05)$. These results suggest that the prefrontal cortex's structure and function both may support $g F$ rather than $g C$. 

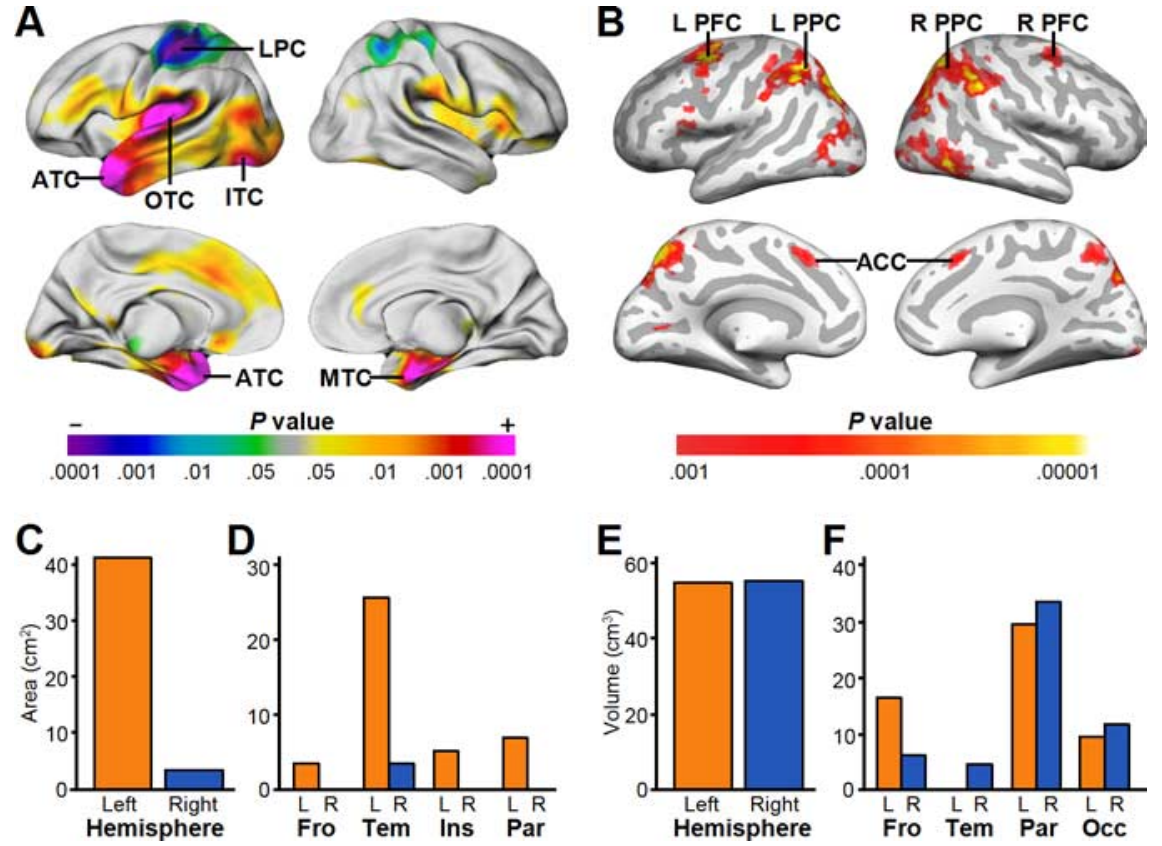

Figure 1. Structural and functional correlates of intelligence and their contrasting laterality. $\boldsymbol{A}$, Correlations between cortical gray matter thickness and WAIS FSIQ. The color bar indicates the statistical significance of the correlations (left, negative correlation; right, positive correlation). Lines point to ROls with high statistical significance ( $p<0.001$ uncorrected): ATC, OTC, ITC, MTC, and LPC. $\boldsymbol{B}$, Correlations between cortical activation level during reasoning tasks and WAIS FSIQ. The color bar indicates the statistical significance of the correlations. Lines point to ROIs with high statistical significance ( $p<0.001$ uncorrected): ACC, PFC, and PPC. $\boldsymbol{C}, \boldsymbol{D}$, Hemispheric $(\boldsymbol{C})$ and lobar $(\boldsymbol{D})$ area sizes of the structural correlates manifesting left dominance. $\boldsymbol{E}, \boldsymbol{F}$, Hemispheric $(\boldsymbol{E})$ and lobar $(\boldsymbol{F})$ volume sizes of the functional correlates demonstrating bilateral symmetry. The sizes of the cortical areas $(\boldsymbol{C}, \boldsymbol{D})$ and the activation clusters $(\boldsymbol{E}, \boldsymbol{F})$ were defined using the correlations of statistical significance ( $p<0.001$ uncorrected). L, Left; $\mathrm{R}$, right; Fro, frontal; Tem, temporal; Ins, insular; Par, parietal; 0cc, occipital lobes.

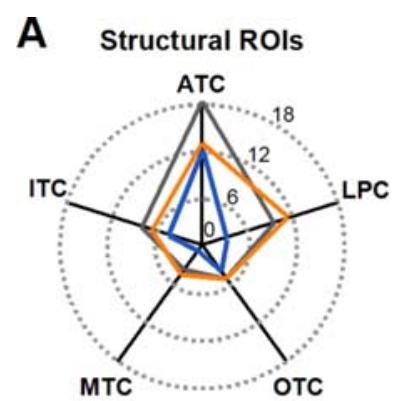

C

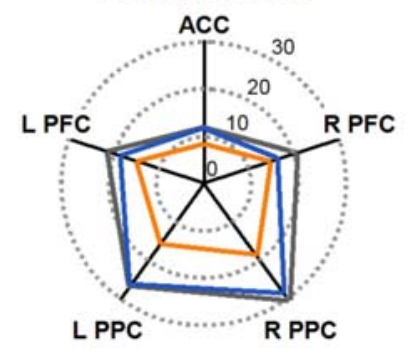

B

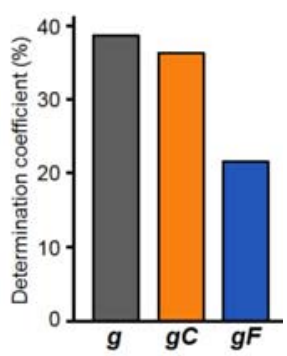

D

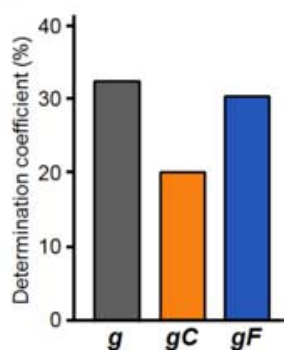

Figure 2. General, crystallized, and fluid intelligence selectively explained by structural and functional ROls. $\boldsymbol{A}, \boldsymbol{C}$, The radar graphs show simple correlations of the cortical thickness $(\boldsymbol{A})$ or peak $t$ score $(\boldsymbol{C})$ of each ROI with the major components of intelligence: $g$ (gray line), $g$ C (orange line), and $g F$ (blue line). $\boldsymbol{B}, \boldsymbol{D}$, The bar graphs display the multiple correlations of all structural $(\boldsymbol{B})$ or functional $(\boldsymbol{D})$ ROI values with the intelligence components. Each bar or line indicates the amount of explained variance $\left(R^{2}\right)$ of individual performance in the intelligence component scores. $g$, Principal component of all WAIS subtests and RPM; $g$ C, principal component of WAIS Verbal Comprehension subtests; $g F$, principal component of WAIS Perceptual Organization subtests and RPM.
Cortical thickness and functional activation selectively explaining $g C$ and $g F$

We hypothesized that the structural and functional correlates reflect different components of $g$. Specifically, the left lateralization suggests that the structural correlates may be more strongly associated with verbal information processing, namely $g C$. We tested for a difference between structural and functional neural correlates of intelligence in their relation to hierarchical components of intelligence, which comprised superordinate $(g)$ and subordinates ( $g C$ and $g F$ ), derived by factor analysis of the RPM and the WAIS subtest scores (see Materials and Methods). $g$ exhibited the highest association with both structural and functional correlates of intelligence (Fig. 2). Gray matter thickness within the target ROIs was more strongly associated with $g C$ than $g F$ (Fig. $2 A$; supplemental Fig. 3, available at www. jneurosci.org as supplemental material). In contrast, neural activity within each node of the frontoparietal network (the functional ROIs) better reflected $g F$ than $g C$ (Fig. 2C; supplemental Fig. 4, available at www.jneurosci.org as supplemental material). Further, multiple linear regression analysis demonstrated that the overall explanatory power of the structural correlates for $g C\left(R^{2}=37 \%\right)$ was greater than for $g F\left(R^{2}=22 \%\right)$, whereas the functional correlates showed a pattern opposite to the structural one ( $g C, R^{2}=20 \%$ vs $g F, R^{2}=$ $31 \%$ ) (Fig. 2B,D). This differential association of two psychological functions $(g F$ and $g C)$ with different brain areas strongly supports our hypothesis. The association between cortical thickness and semantic knowledge was further demonstrated by higher correlations for WAIS subtest scores based on verbal rather than nonverbal (performance) tests (supplemental Table 3 , available at www.jneurosci.org as supplemental material).

\section{Intellectual category's impact on cortical thickness differences among IQ levels}

The correlational coefficients and intelligence factors, however, are too simple figures abstracted from a given dataset. They can scarcely give a close-up view on the practical relation between psychometric intelligence test scores (IQ) and the cortical gray matter thickness (in millimeters). To resolve this uncertainty, we divided the sample into three groups of virtually equal numbers (i.e., superior IQ, high IQ, and average IQ groups) based on their conventional scales on four domains of intelligence tests, WAIS verbal IQ (VIQ), performance IQ (PIQ), FSIQ, and RPM scores (supplemental Table 4, available at www.jneurosci.org as supplemental material), and determined differences in the cortical thickness between the IQ groups (Fig. 3). Regardless of the intellectual criteria, the most significant cortical differences occurred in the left temporal cortex (LTC) across the cerebrum. In regard to test score, VIQ differences had the strongest association with cortical thickness, particularly in the LTC. This indicates that cortical thickness in the temporal cortex primarily reflects verbal rather than fluid ability. Notably, the relation between cortical 

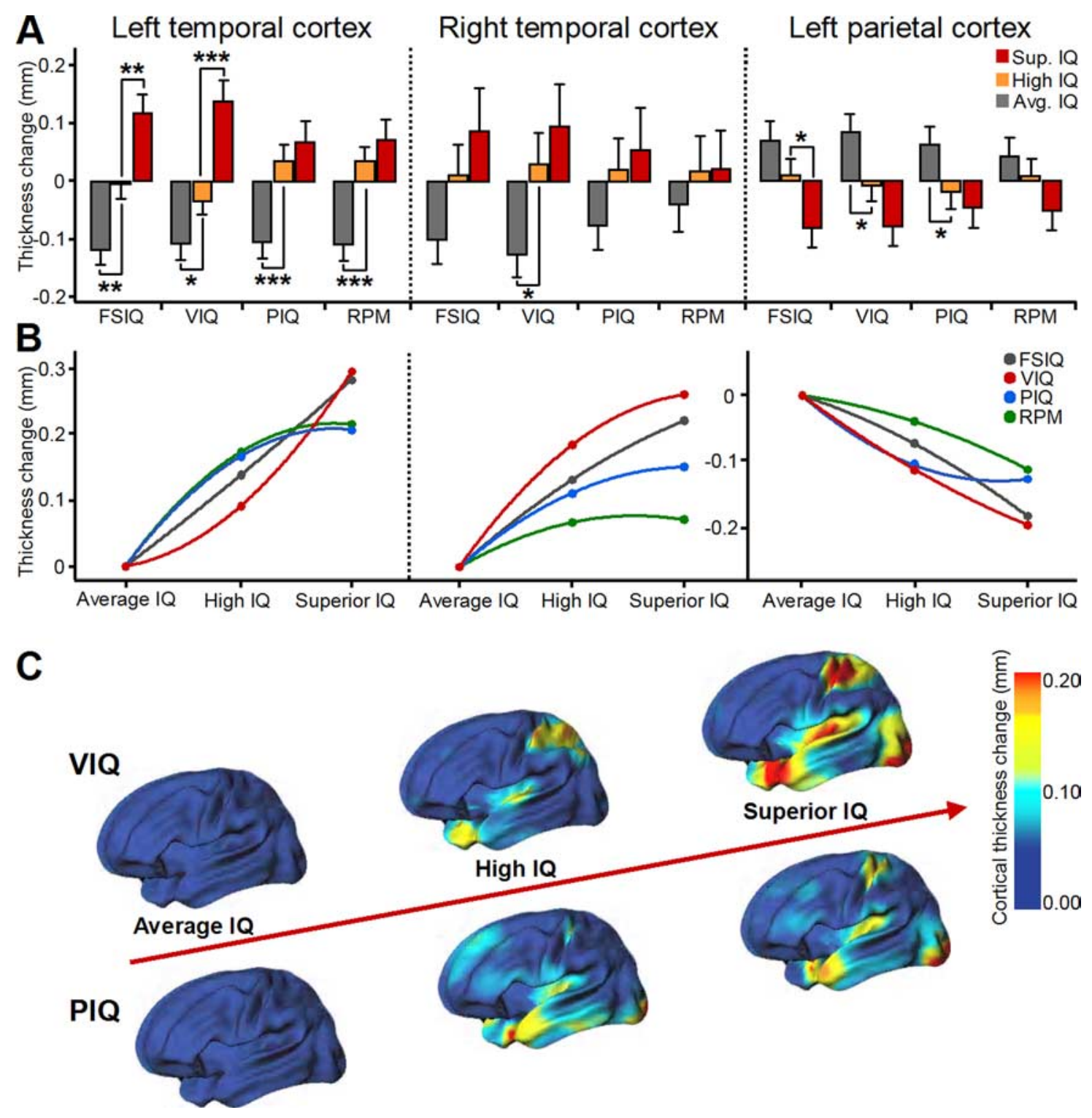

Figure 3. Intellectual domain effects on cortical thickness changes as a function of $\mathrm{IQ}$ level. $\boldsymbol{A}$, Cortical thickness differences between adjoining levels of $\mathrm{IQ}$ as affected by intelligence criteria and brain lobes. The superior, high, and average IQ groups were evenly divided according to four intelligence criteria, $\mathrm{FSIQ}, \mathrm{VIQ}, \mathrm{PIQ}$, and RPM scores. The cortical thickness of each lobe is represented by the averaged value of all ROls within the lobe. Sup., Superior; Avg., average. ${ }^{*} p<0.05$; ${ }^{* *} p<0.01$; ${ }^{* * *} p<0.001$, two-tailed $t$ test. $B$, C, Cortical thickness deviations from the thickness of the average IQ group used as zero reference. VIQ groups are better described by a linear or quadratic function, whereas PIQ groups are better described by a logarithmic one. The brain maps show absolute thickness changes at each cortical point, based on VIQ and PIQ levels.

thickness and VIQ was close to linear between the groups and even increased sharply in the superior IQ range for the LTC. However, differences in PIQ and RPM were less in the superior IQ range compared with the average range. These findings suggest a possible biological basis for Spearman's "law of diminishing returns," which predicts that acquired knowledge and educationally relevant information associated with $g C$ become more salient than purer measures of $g$ among subjects of higher intelligence (Jensen, 1998).

\section{Neurometric IQ model: predicting psychometric IQ from neurobiological measures}

We validated our findings by testing a neurometric model of intelligence that we developed prospectively. Specifically, we postulated that combining structural and functional neural correlates of intelligence would allow us to formulate a model capable of accurately predicting psychometric intelligence in an indepen- dent sample (i.e., prospective prediction, rather than fitting a model post hoc to a dataset). The model combining structural and functional measures predicted IQ strongly in the test sample $(R=0.71 ; p<0.001)$ (Fig. 4). Moreover, functional predictors contributed above structural, and vice versa, reiterating our finding of a differential association of psychological function with brain regions. As a stringent validation of the model, we computed an IQ estimate for each test-sample participant. Even using not free parameters, but fixed parameters ( $\alpha$ and $\beta$ values derived from the model pools), estimated IQ predicted IQ strongly ( $r=$ 0.67; $p<0.001$ ) (Fig. 4B), not differing statistically from the benchmark level of predictability (IQ from RPM, difference between dependent correlations, $Z=1.06$; $p=0.29$ ). Thus, within this population, MR-derived brain images can be used to predict intelligence to an impressive degree. More critically for the present purposes, both structure and function made distinct contributions to the model. 
A Structural predictor $(n=116) B$
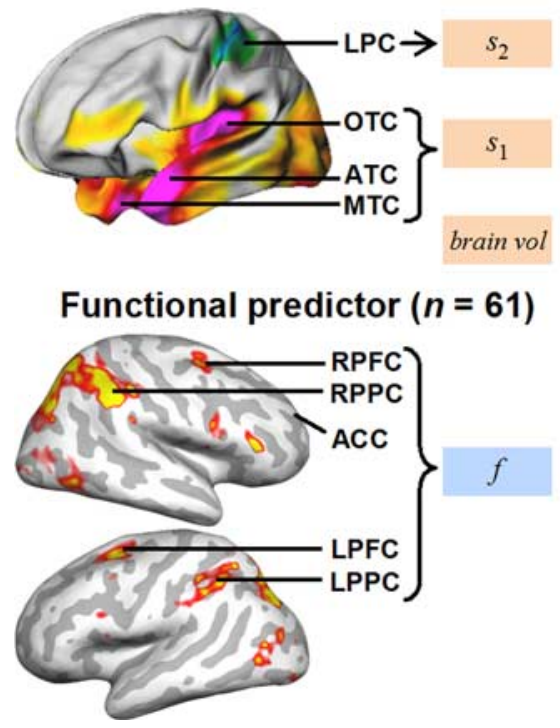

Figure 4. Prediction of psychometric IQ from neurobiological measures. $A$, Specification of a neurometric IQ model. To specify potential predictors, we correlated IQ with cortical thickness (top) or activation level (bottom) at each voxel, and thresholded $(p<0.001)$ to define ROls. We reduced redundancy by averaging ROls that covaried $(p<0.001)$, leaving brain volume and three ROI-based candidate predictor variables. Interaction terms allowed sex to moderate the relation between the MR-based variables and IQ. To specify a model, we screened potential predictors by regressing IQ on them simultaneously, retaining five as the model. $\boldsymbol{B}$, Test of the model. We estimated IQ using the model in an independent sample. Estimated IQ correlated with measured IQ, indicating successful prediction. For the strict model (fixed parameters), parameter values were obtained by fitting the model (regression) in the datasets for model specification.

\section{Discussion}

To our knowledge, the present study describes the first simultaneous investigation of structural and functional correlates of intelligence in a large sample. We found strong support for the hypothesis that spatially distributed and functionally distinct brain regions contribute to intelligence, which is strong evidence against a single-locus or single-network account of the neural bases of intelligence. Cortical gray matter thickness covaried more strongly with $g C$, whereas functional activity during a fluid reasoning task covaried more strongly with $g F$. By combining two brain imaging modalities into a single study, our finding builds on previous work showing neural correlates of intelligence in a single modality (Duncan et al., 2000; Frangou et al., 2004; Gong et al., 2005; Gray et al., 2003; Haier et al., 2004; Lee et al., 2006). It is also the first demonstration of a prediction of intelligence from brain structure and function alone (cf. Deary et al., 2007). Moreover, by building and validating a neurometric model that accurately predicts IQ scores in an independent sample, we have established the robustness of our finding, as well as a new benchmark for neural models of intelligence.

One difference between our structural and functional findings, aside from the differentiable relations to $g C$ and $g F$, was that that the structural correlates were predominantly localized in the left hemisphere, whereas the functional correlates were symmetrically distributed. This contrast is consistent with findings from studies of commissurotomy patients showing that the left hemisphere is specialized for verbal expression and numerical computation (Gazzaniga and Sperry, 1967) but neither hemisphere is specialized for fluid reasoning skills assessed by the RPM (Zaidel et al., 1981). With the contrasting laterality, our finding that $g C$ and $g F$ relate differentially to brain structure and function further supports a neural basis for the crystallized-fluid distinction within $g$ (Cattell, 1943). Of course, structure influences function, and vice versa, which is likely why both subfactors of $g$ were related to both structure and function despite stronger associations with one modality.

In our study, $g C$ was associated with greater cortical thickness in regions of the temporal lobe and surrounding areas that may be involved in processing semantic information (McClelland and Rogers, 2003). Semantic information about an object may be stored close to the brain regions dedicated to that type of information. Specifically, sound and image of an object recruit opercular and inferior temporal cortices, respectively (Martin et al., 1995; Kellenbach et al., 2001). Temporal pole is thought to be responsible for integrating diverse semantic information from distinct brain regions. Studies of patients with semantic dementia implicate the anterior temporal lobe in semantic working memory as well as memory storage (Hodges et al., 1992; Mummery et al., 1999; Gainotti, 2006). In monkeys, structural reorganization of cortical neurons, including synapse formation and axonal dendritic growth, tracks with consolidation of semantic-like memory traces in temporal cortex (Tokuyama et al., 2000;

Chklovskii et al., 2004).

Human opercular temporal cortex or planum temporale is demonstrated to be anatomically and functionally lateralized on the left hemisphere (Geschwind and Levitsky, 1968; Devlin et al., 2003). Because the region contains primary and associative auditory cortex and the posterior part constitutes Wernicke's area, these asymmetries are often cited as the anatomical basis of the left hemisphere dominance for language. In our results, whereas the anterior temporal cortex was associated with all the verbal subtest scores, the opercular temporal cortex was correlated only with the two verbal scores on Information and Comprehension subtests $(p<0.01)$ (supplemental Table 3, available at www. jneurosci.org as supplemental material). The two tests, measuring fund of factual information and common sense, are strongly influenced by culture and education. Thus, the opercular temporal cortex might be more specialized for storing and retrieving verbal knowledge on the culture, compared with the anterior temporal cortex possibly having general functions such as semantic integration and working memory.

In summary, individual differences in $g C$ may depend on declarative knowledge stored in the temporal lobe and association areas that are the product of task-relevant neural connections and that manifest as structural differences across individuals. However, previous neuroimaging studies investigating structural correlates of intelligence did not tend to implicate temporal regions. This discrepancy may arise from technical limitations of voxelbased morphometry (Bookstein, 2001; Mehta et al., 2003) or unreliability in their normalization procedures [in particular, the temporal lobe's discrepancy between the Montreal Neurological Institute template and the Talairach brain extends to $\sim 10 \mathrm{~mm}$ (Westbury et al., 1999; Brett et al., 2002)].

$g F$ was associated with increased brain activity in a frontopa- 
rietal network involved in working memory and executive control of behavior. This finding is consistent with previous research demonstrating the role of this network in supporting $g$ (Gray et al., 2003; Lee et al., 2006). In contrast, neuroanatomical work has not reached a consensus about the substrate of $g$ (Frangou et al., 2004; Gong et al., 2005), and it is notable that few anatomical studies have strongly implicated specific areas in $g$ (Jung and Haier, 2007). A first possible reason is that the structural correlates of intelligence change with age (Shaw et al., 2006). In particular, the frontal and parietal regions decline in gray matter density more rapidly than the temporal region (Sowell et al., 2003). Studies of brain structure might be more susceptible to subject demographics (age mean and variation). For crosssectional studies, it may be crucial to select subjects reaching a period of developmental stability or to stratify by age. Second, the type of intelligence measure might affect the structural findings more severely than expected. Although a short form of WAIS is highly correlated with the full version, the short version increases measurement error (Brooks and Weaver, 2005). Shaw et al. (2006) show relatively strong correlations in the prefrontal regions in the young adult map comparable with our WAIS map. They used a short form consisting of only four subtests, one of which is matrix reasoning. Intriguingly, our RPM (matrix reasoning) map showed stronger correlation in prefrontal region than our WAIS map, and seemed more similar to their map.

By more clearly defining a neural basis for the distinction between $g C$ and $g F$, and establishing a new benchmark for prediction of intelligence based on brain images, our findings present a new challenge for other hierarchical models of $g$ (Johnson and Bouchard, 2005) that strive for both theoretical strength and mechanistic plausibility. Neurometric models based on these alternative conceptualizations of $g$ need not only to generate similarly differentiable relations between their components and brain structure and function, but also must yield comparable or greater predictive power.

Our neurometric model of IQ contributes to a literature showing that brain images can be used to predict complex behaviors and traits (Haynes and Rees, 2006; Knutson et al., 2007). Although our model still does not approach the predictive power of psychometric tests, its high accuracy suggests that neurometric assessments of intelligence may soon become a useful complement to psychometric test. For example, brain images might be used to improve intelligence estimates for individuals whose psychometric scores systematically underestimate their IQ. We hope that future research will build on our neurometric model of intelligence, both refining it so that it generalizes to other populations and expanding it to enhance its accuracy.

\section{References}

Bookstein FL (2001) "Voxel-based morphometry" should not be used with imperfectly registered images. Neuroimage 14:1454-1462.

Brett M, Johnsrude IS, Owen AM (2002) The problem of functional localization in the human brain. Nat Rev Neurosci 3:243-249.

Brooks BL, Weaver LE (2005) Concurrent validity of WAIS-III short forms in a geriatric sample with suspected dementia: verbal, performance and full scale IQ scores. Arch Clin Neuropsychol 20:1043-1051.

Carroll JB (1993) Human cognitive abilities: a survey of factor-analytic studies. New York: Cambridge UP.

Cattell RB (1943) The measurement of adult intelligence. Psychol Bull 40:153-193.

Chabris CF (2006) Cognitive and neurobiological mechanisms of the law of general intelligence. In: Integrating the mind: domain general versus domain specific processes in higher cognition (Roberts MJ, ed). Hove, UK: Psychology.
Chklovskii DB, Mel BW, Svoboda K (2004) Cortical rewiring and information storage. Nature 431:782-788.

Craik FI, Bialystok E (2006) Cognition through the lifespan: mechanisms of change. Trends Cogn Sci 10:131-138.

Deary IJ, Ferguson KJ, Bastin ME, Barrow GWS, Reid LM, Seckl JR, Wardlaw JM, MacLullich AMJ (2007) Skull size and intelligence, and King Robert Bruce's IQ. Intelligence 35:519-525.

Devlin JT, Raley J, Tunbridge E, Lanary K, Floyer-Lea A, Narain C, Cohen I, Behrens T, Jezzard P, Matthews PM, Moore DR (2003) Functional asymmetry for auditory processing in human primary auditory cortex. J Neurosci 23:11516-11522.

Duncan J, Emslie H, Williams P, Johnson R, Freer C (1996) Intelligence and the frontal lobe: the organization of goal-directed behavior. Cognit Psychol 30:257-303.

Duncan J, Seitz RJ, Kolodny J, Bor D, Herzog H, Ahmed A, Newell FN, Emslie H (2000) A neural basis for general intelligence. Science 289:457-460.

Engle RW, Tuholski SW, Laughlin JE, Conway AR (1999) Working memory, short-term memory, and general fluid intelligence: a latent-variable approach. J Exp Psychol Gen 128:309-331.

Frangou S, Chitins X, Williams SC (2004) Mapping IQ and gray matter density in healthy young people. Neuroimage 23:800-805.

Gainotti G (2006) Anatomical functional and cognitive determinants of semantic memory disorders. Neurosci Biobehav Rev 30:577-594.

Gazzaniga MS, Sperry RW (1967) Language after section of the cerebral commissures. Brain 90:131-148.

Geschwind N, Levitsky W (1968) Human brain: left-right asymmetries in temporal speech region. Science 161:186-187.

Gong QY, Sluming V, Mayes A, Keller S, Barrick T, Cezayirli E, Roberts N (2005) Voxel-based morphometry and stereology provide convergent evidence of the importance of medial prefrontal cortex for fluid intelligence in healthy adults. Neuroimage 25:1175-1186.

Gray JR, Thompson PM (2004) Neurobiology of intelligence: science and ethics. Nat Rev Neurosci 5:471-482.

Gray JR, Chabris CF, Braver TS (2003) Neural mechanisms of general fluid intelligence. Nat Neurosci 6:316-322.

Haier RJ, Jung RE, Yeo RA, Head K, Alkire MT (2004) Structural brain variation and general intelligence. Neuroimage 23:425-433.

Haynes JD, Rees G (2006) Decoding mental states from brain activity in humans. Nat Rev Neurosci 7:523-534.

Hodges JR, Patterson K, Oxbury S, Funnell E (1992) Semantic dementia: progressive fluent aphasia with temporal lobe atrophy. Brain 115:1783-1806.

Jensen AR (1998) The g factor: The science of mental ability. Westport, CT: Praeger.

Johnson W, Bouchard TJ (2005) The structure of human intelligence: it is verbal, perceptual, and image rotation (VPR), not fluid and crystallized. Intelligence 33:393-416.

Jung RE, Haier RJ (2007) The parieto-frontal integration theory (P-FIT) of intelligence: converging neuroimaging evidence. Behav Brain Sci 30:135154; discussion 154-187.

Kellenbach ML, Brett M, Patterson K (2001) Large, colorful, or noisy? Attribute- and modality-specific activations during retrieval of perceptual attribute knowledge. Cogn Affect Behav Neurosci 1:207-221.

Kim JS, Singh V, Lee JK, Lerch J, Ad-Dab'bagh Y, MacDonald D, Lee JM, Kim SI, Evans AC (2005) Automated 3-D extraction and evaluation of the inner and outer cortical surfaces using a Laplacian map and partial volume effect classification. Neuroimage 27:210-221.

Knutson B, Rick S, Wimmer GE, Prelec D, Loewenstein G (2007) Neural predictors of purchases. Neuron 53:147-156.

Kovas Y, Plomin R (2006) Generalist genes: implications for the cognitive sciences. Trends Cogn Sci 10:198-203.

Lee H, Devlin JT, Shakeshaft C, Stewart LH, Brennan A, Glensman J, Pitcher K, Crinion J, Mechelli A, Frackowiak RS, Green DW, Price CJ (2007) Anatomical traces of vocabulary acquisition in the adolescent brain. J Neurosci 27:1184-1189.

Lee KH, Choi YY, Gray JR, Cho SH, Chae JH, Lee S, Kim K (2006) Neural correlates of superior intelligence: stronger recruitment of posterior parietal cortex. Neuroimage 29:578-586.

Martin A, Haxby JV, Lalonde FM, Wiggs CL, Ungerleider LG (1995) Discrete cortical regions associated with knowledge of color and knowledge of action. Science 270:102-105. 
McClelland JL, Rogers TT (2003) The parallel distributed processing approach to semantic cognition. Nat Rev Neurosci 4:310-322.

Mehta S, Grabowski TJ, Trivedi Y, Damasio H (2003) Evaluation of voxelbased morphometry for focal lesion detection in individuals. Neuroimage 20:1438-1454.

Mummery CJ, Patterson K, Wise RJ, Vandenberghe R, Price CJ, Hodges JR (1999) Disrupted temporal lobe connections in semantic dementia. Brain 122:61-73.

Shaw P, Greenstein D, Lerch J, Clasen L, Lenroot R, Gogtay N, Evans A, Rapoport J, Giedd J (2006) Intellectual ability and cortical development in children and adolescents. Nature 440:676-679.

Sowell ER, Peterson BS, Thompson PM, Welcome SE, Henkenius AL, Toga AW (2003) Mapping cortical change across the human life span. Nat Neurosci 6:309-315.

Spearman C (1904) General intelligence, objectively determined and measured. Am J Psychol 15:201-293.
Thurstone LL (1940) The vectors of the mind. Chicago: University of Chicago.

Tokuyama W, Okuno H, Hashimoto T, Xin Li Y, Miyashita Y (2000) BDNF upregulation during declarative memory formation in monkey inferior temporal cortex. Nat Neurosci 3:1134-1142.

Tranel D, Manzel K, Anderson SW (2008) Is the prefrontal cortex important for fluid intelligence? A neuropsychological study using matrix reasoning. Clin Neuropsychol 22:242-261.

Waltz JA, Knowlton BJ, Holyoak KJ, Boone KB, Mishkin FS, de Menezes Santos M, Thomas CR, Miller BL (1999) A system for relational reasoning in human prefrontal cortex. Psychol Sci 10:119-125.

Westbury CF, Zatorre RJ, Evans AC (1999) Quantifying variability in the planum temporale: a probability map. Cereb Cortex 9:392-405.

Zaidel E, Zaidel DW, Sperry RW (1981) Left and right intelligence: case studies of Raven's progressive matrices following brain bisection and hemidecortication. Cortex 17:167-185. 